BNL --45923

DE9 I 009842

\title{
Transition Crossing in the RHIC
}

\author{
Jie Wei
}

March 1991

DISCLAIMER

This report was prepared as an account of worl sponsored by an agency of the United States Government. Neither the United States Government nor any agency thereof, nor any of their employees, makes any warranty, express or implied, or assumes any legal liability or responsibility for the accuracy, completeness, or usefulness of any information, apparatus, product, or process disclosed, or represents that its use would not infringe privately owned rights. Reference herein to any specific commercial product, process, or service by trade name, trademark, manufacturer, or otherwise does not necessarily constitute or imply its endorsement, recommendation, or favoring by the United States Government or any agency thereof. The views and opinions of authors expressed herein do not necessarily state or reflect those of the United States Government or any agency thereof.

\section{RH I C P R O E C T}

\author{
Brookhaven National Laboratory \\ Associated Universities, Inc. \\ Upton, NY 11973
}

Under Contract No. DE-AC02-76CH-00016 with the UNITED STATES DEPARTMENT OF ENERGY 


\title{
Transition Crossing in the RHIC
}

\author{
JIE WEI \\ Accelerator Development Dcpartment \\ Brookhaven National Laboratory \\ Upton, New York 11979
}

\begin{abstract}
This report summarizes the study of various longitudinal problems pertaining to the transition-energy crossing in the proposed Relativistic IIeavy Ion Collider. Scaling laws are provided for the effects of chromatic non-linearity, self-field mismatch, and microwave instability. It is indicated that the beam loss and bunch-area growilh are mainly caused by the chromatic non-linear effect, which is enhanced by the space-charge force near transition. Computer simulation using the program TIBETAN shows that a " $\gamma_{\boldsymbol{x}}$-jump" of about 0.8 unit within a time period of $60 \mathrm{~ms}$ is adequate to achieve a "clean" crossing, provided that the remnant voltage of the $160 \mathrm{MHz}$ rf system is less than $10 \mathrm{kV}$.
\end{abstract}




\section{Introduction}

The transition-energy crossing of charged particles is characterized by a time scale $T_{c}$ during which the particle motion is non-adiabatic, ${ }^{1-4}$

$$
T_{c}=\left(\frac{\pi A E_{0} \beta_{a}^{2} \gamma_{T}^{3}}{q e \hat{V}\left|\cos \phi_{a}\right| \dot{\gamma}_{s} h \omega_{0}^{2}}\right)^{\frac{1}{3}},
$$

where the subscript $s$ represents the synchronous value, and,

$$
\begin{aligned}
& q e=\text { electric charge carried by the particle } \\
& \hat{V}=\text { peak voltage of the } \mathrm{r} \text { accelerating system } \\
& h=\text { harmonic number of the rf accelerating systein } \\
& \eta_{0}=1 / \gamma_{T}^{2}-1 / \gamma_{s}^{2} \\
& \gamma_{T}=\text { transition energy } \\
& \phi_{s}=\text { synchronous phase } \\
& \omega_{s}=\text { synchronous revolution frequency } \\
& \beta_{s} c=\text { synchronous velocity }
\end{aligned}
$$$$
A=\text { atomic mass number of the particle }
$$$$
A E_{0}=A m_{0} c^{2} \gamma_{s}, \text { synchronous energy of the particle. }
$$

Problems related to Lransition crossing can mainly be divided into two calegories: singleand multi-particle. In the former category, we study the effect of chromatic non-linearities which impel particles of different momenta to cross transition at different times; while in the latter, we study the bunch-shape mismatch and microwave instability induced by low-and liglt-frequency self fields, respectively. Theoretical estimates are presented in the first part of section 2; results of computer simulation are addressed in the second part. Compensation methods and requirements are discussed in section 3 . With the given $\gamma_{T}$-jump, a tolerance is provided in section 4 to the remnant voltage of the $100 \mathrm{MHz}$ rf system.

\section{Problems at Transition Energy}

In this section, scaling laws are obtained for the effects of chromatic non-linearity, selffield mismatch, and microwave instability at transition. In the alssence of a $\gamma_{T}$-jump, the 
growth in bunch area due to these effects is estimated.

\subsection{Theoretical Estimates}

\section{A. Cliromatic non-linear effect}

Particles of different momenta traverse closed orbits of different lengths $L$. The difference may be expressed in terms of the momentum deviation $(\delta \equiv \Delta p / p)$ as

$$
\frac{L}{L_{\varepsilon}}=1+\frac{\delta}{\gamma_{T}^{2}}\left[1+\alpha_{1} \delta+O\left(\delta^{2}\right)\right]
$$

The so-called "frequency-slip factor" $\eta$ can thus be written as

$$
\eta=\eta_{0}+\eta_{1} \delta+\cdots
$$

where

$$
\eta_{0}=\frac{1}{\gamma_{T}^{2}}-\frac{1}{\gamma_{0}^{2}}, \quad \text { and } \quad \eta_{1} \approx \frac{2}{\gamma_{0}^{2}}\left(\alpha_{1}+\frac{3 \beta_{s}^{2}}{2}\right) .
$$

The two terms in $\eta_{1}$ correspond respectively to the differences in circumference and velocity for particles of different momenta at the first non-linear order. The effect of $\eta_{1}$ on the particle notion is important only near the transition energy when $\eta_{0}$ approaches zero. Define $^{4-6}$ the "non-linear tine" $T_{n l}$ during which $\left|\eta_{1} \hat{\delta}\left(\gamma_{T}\right)\right|$ is larger than $\left|\eta_{0}\right|$,

$$
T_{r l}=\frac{\left|\left(\alpha_{1}+\frac{3}{2} \beta_{s}^{2}\right)\right| \hat{\delta}\left(\gamma_{T}\right) \gamma_{T}}{\dot{\gamma}_{s}}
$$

where

$$
\hat{\delta}\left(\gamma_{\gamma}\right)=\frac{2^{1 / 2} \omega_{s}\left(h S q e \hat{V}\left|\cos \phi_{s}\right| T_{c}\right)^{1 / 2}}{3^{2 / 3} \pi^{1 / 2} \Gamma(2 / 3) A E_{0} \beta_{s}^{2}}
$$

is the maxinum momentum spread at transition, $\Gamma(2 / 3) \approx 1.354$, and $S$ is the bunch area before transition. The effective increase in the bunch area during the crossing depends on the ratio of $T_{n l}$ to $T_{\mathrm{c}}$ (eqs. 4.25 and $4.27 \mathrm{in}$ ref.4),

$$
\frac{\Delta S}{S} \approx \begin{cases}0.76 \frac{T_{n l}}{T_{c}}, & \text { for } T_{n l} \ll T_{c} ; \\ e^{\frac{1}{3}\left(\frac{T_{n l}}{T_{c}}\right)^{3 / 2}}-1, & \text { for } T_{n l} \geq T_{c}\end{cases}
$$


Bean loss occurs if the effective bunch area $S+\Delta S$ after transition is larger than the bucket area.

It is assumed ${ }^{7}$ for the RHIC that $\gamma_{T}=24.7, \omega_{x}=4.91 \times 10^{5} \mathrm{~s}^{-1}, h=342, \hat{V}=300 \mathrm{kV}$, $\phi_{s}=0.161$ rad., and $\alpha_{1}=0.6$. With these paraneters, it can be evaluated that $\dot{\gamma}_{\mathrm{s}}=1.6$ $\mathrm{s}^{-1}, T_{c}=41 \mathrm{~ms}, \hat{\delta}\left(\gamma_{T}\right)=4.3 \times 10^{-3}$, and $T_{n l}=139 \mathrm{~ms}$. According to eq. 4 , the phase-space area enclosed by the trajectory of the particles near the edge of the bunch after transition is much larger than the bucket area. Therefore, beam loss is expected to occur in the absence of a $\gamma_{T}$-jump. Quantitatively, the amount of beam loss depends on the particle distribution in the phase space.

\section{B. Bunch-shape mismatch}

Both reaclive and resistive impedances cause mismatch ${ }^{8-9}$ in the nominal bunch shape at the time the synchronous phase is jumped at transition.

A reactive impedance changes the focusing force of the rf system differently below and above transition. The amount of mismatch is then proportional to the ratio of the self field to the rf field provided by the accelerating cavilies. For a parabolic distribution, the effective increase in the bunch area due to the mismatch, induced by a coupling inpedance $\left|Z_{L} / n\right|$ at low frequency range, is (eq. 5.18 in ref.4)

$$
\frac{\Delta S}{S}=\frac{2 h \hat{I}\left(\gamma_{T}\right)\left|Z_{L} / n\right|}{\hat{V}\left|\cos \phi_{J}\right| \hat{\phi}^{2}\left(\gamma_{T}\right)}
$$

where

$$
\hat{I}=\frac{3 h N_{0} q e \omega_{s}}{4 \hat{\phi}}
$$

is the peak current, and

$$
\hat{\phi}\left(\gamma_{T}\right)=3^{1 / 6} \Gamma(2 / 3)\left(\frac{2 h S}{\pi q e^{\hat{V^{\gamma}}}\left|\cos \phi_{a}\right| T_{c}}\right)^{1 / 2}
$$

is the maximum phase spread of the bunch at transition.

The effective impedance of the space charge below the cutoff frequency is

$$
\frac{Z}{n}=\frac{i g_{0} Z_{0}}{2 \beta, \gamma_{f}^{2}},
$$


where $g_{0}$ is a geometric factor, and $Z_{0}=\left(\varepsilon_{0} c\right)^{-1}=377 \Omega$. Taking $g_{0}=4.5$, this corresponds to a capacitive impedance of about $1.2 \Omega$ at transilion. With an intensity of $N_{0}=1 \times 10^{9}$ ${ }^{197} \mathrm{Au}^{79+}$ ions per bunch, the increase of bunch area due to the corresponding bunch-shape mismatch is about $60 \%$ in the absence of a $\gamma_{T}$-junp.

In addition to the mismatch, a resistive impedance causes energy dissipation which partly cancels the energy gain from the $\mathrm{r} f$ acceleration. Because this cancellation induces a shift in the synchronous phase $\left(\phi_{s}\right)$, the amount of synchronous phase $\left(\pi-2 \phi_{s}\right)$ to be junped at transition is changed accordingly. If the resistive impedance al low frequency is $R$, the shift in synchronous phase can also be shown as (eq. 5.22 in ref.4)

$$
\Delta \phi_{s}=\frac{\hat{I} \mathcal{R}}{\hat{V}\left|\cos \phi_{s}\right|}
$$

for $\Delta \phi$, to be much smaller than 1. Compared with the mismatch due to the space charge, the eflect of the resistive impedance is estimated to be small for the transition crossing in the RIIIC.

\section{Microwave instability}

Near the transition energy, the revolution-frequency spread which provides Landau damping vanishes along with the vanishing phase stability and the decreasing synchrotronoscillation frequency. Both the reactive and the resistive conponents of the coupling impedance are likely to induce an instability. However, since particles cross transition with a non-zero acceleration rate, the synchrotron-oscillation frequency defined by the time derivative of the angle variable (canonically conjugate with the action variable) of the system IIamiltonian, is also non-zero at transition. ${ }^{10}$ Consequently, the threshold for microwave instability to occur at transition is (eq. $5.58 \mathrm{in}$ ref.4) for the parabolic distribution

$$
D_{0} \equiv \frac{8 h \hat{I}\left(\gamma_{T}\right)\left|Z_{H} / n\right|}{3 \hat{V}\left|\cos \phi_{A}\right| \hat{\phi}^{2}\left(\gamma_{T}\right)} \geq 1
$$

where $\left|Z_{H} / n\right|$ refers to the coupling inpedance at microwave frequency range. Again, the coefficient in eq. 8 may differ for different particle distribution. 
In the case that the beam current is below the threshold (Eq. 8), the bunch crosses the transition without experiencing microwave instability. On the other hand, in the case that this threshold is exceeded, the time period $T_{m w}$ during which the instability occurs can be estimated, ${ }^{10}$

$$
T_{n \omega w} \approx \frac{2 \pi}{3^{5 / 6} \Gamma^{2}(2 / 3)}\left(D_{\mathrm{s}}-1\right) T_{\mathrm{c}} \approx 1.37\left(D_{0}-1\right) T_{\mathrm{c}}, \quad \text { for } D_{0}-1 \ll 1 .
$$

The amount of growth of the density amplitude, which is defined as the ratio of the amplitude increment of the density aisturbance at the end, to the anplitude at the beginning of the time interval $T_{m w}$, is found to be

$$
\frac{\sqrt{3} n}{4 h} \hat{\phi}\left(\gamma_{T}\right)\left(D_{0}-1\right)^{2}
$$

With a beam intensity of $1 \times 10^{9}$ ions per bunch and a capacilive impedance of $1.2 \Omega$ due to the space charge, it can be calculated that $D_{0} \approx 0.9$. Thereiore, in the absence of a $\gamma_{T}$-jump the bean is close to the microwave-instability threshold.

The theoretical estimates indicale that the primary concern at transition is due to the chromatic non-linear effect. The development of the "non-linear tails" after the synchronousphase jump is further enlanced by the space-charge force. Computer simulation is needed to understand more precisely the various mechanisms and to quantitatively determine the crossing efficiency.

\subsection{Results of computer simulation}

The computer program TIBETAN has been ${ }^{4}$ used to study the transition-crossing process in the RIIIC. The program simulales the longitudinal motion of a particle bean by tracking a collection of macro-particles in phase space. It constructs the self lields directly in the time (phase) domain. The bin length used for the construction of the self fields is cliosen in accordance with the cutoff frequency of the vacuum pipe. Beam-induced fields are calculated every turn with 3600 macro-parlicles. Before transition, the bunch of ${ }^{197} \mathrm{Au}^{79+}$ ions is assumed to have a truncated Gaussian-like distribution in longitudinal phase space 
with an area of $0.3 \mathrm{eV} \cdot \mathrm{s} / \mathrm{u}$. With $\alpha_{1}=0.6$ and $10^{9}$ ions per bunch, the chromatic nonlinear effect, enlanced by the self-field mismatch, results in a beam loss of about $70 \%$ in the alssence of a $\gamma_{T}$-jump.

\section{Crossing Transition with a $\gamma_{T^{-J}}$ ump}

An effective way to cure both the beam-induced and the chromatic non-linear effect is to increase the transition-crossing rate of the beam. This can be accomplished either by temporarily adjusting the lattice to achieve a $\gamma_{T}$-jump, or by nanipulating the synchronous phase to achieve a larger acceleration rate. Previous studies, ${ }^{4,11}$ however, indicate that the latter results in a mismatch at transition which is not negligible.

The method of $\gamma_{T}$-jump, which has been successfully used in many accelerators, provides a large crossing-rate enlancement without causing severe mismatch at transilion. In the case that the non-linear effect is dominant, the amount $\Delta \gamma_{T}$ of jump needed to climinate the un-desired beam loss and bunch-area growth is ${ }^{10,12}$

$$
\Delta \gamma_{T} \approx 4 \dot{\gamma}_{s} T_{n l}
$$

with both $\dot{\gamma}$, and $T_{n l}$ taking the original values; in the case that the self-ficld effect is doninant, the amount of jump should satisfy

$$
\frac{\Delta S}{S}=\left.\frac{2 h \hat{I}(\gamma)\left|Z_{L_{t} H} / n\right|}{\hat{V}\left|\cos \phi_{s}\right| \hat{\phi}^{2}(\gamma)}\right|_{\gamma=\gamma_{T} \pm \Delta \gamma_{T} / 2} \ll 1,
$$

where $\gamma_{T} \pm \Delta \gamma_{T} / 2$ correspond to the instants just after and before the $\gamma_{T}$-jump, $\hat{I}$ is given by Eq. 6 , and the phase spread at the adiabatic regime is

$$
\hat{\phi}=S^{1 / 2}\left(\frac{-2 h^{3} \omega_{s}^{2} \eta_{0}}{\pi \lambda E_{0} \beta_{a}^{2} q e \hat{V} \cos \phi_{s}}\right)^{1 / 4} .
$$

Furthernore, the jump should be accomplished in a short time period so that the effective crossing rate is much larger than the original acceleration rale. .

It is oblained that a jump of 0.8 unit performed in about 60 ins is required to elininate the chronalic non-linear effect. With such a jump, the growth in the bunch area due to 
the self-field mismatch is about $10 \%$ (Eq. 12). Fig. 1 shows the longitudinal phase-space diagrams before, at, and after transition at $\gamma_{s}=24.5,24.7$, and 24.9 , respectively. The transition is crossed without beam loss.

Fig. 2 shows the longitudinal bunch area $S_{f}=S+\Delta S$ after transition as a function of the bunch area $S$ before transition. For a bunch area $S$ smaller than $0.3 \mathrm{eV} \cdot \mathrm{s} / \mathrm{u}$, the effect of the non-linearity is reduced, while that of the self-field nismatch and microwave instability becomes dominant (Fig. 3). On the other hand, for an area larger than 0.3 $\mathrm{eV} \cdot \mathrm{s} / \mathrm{u}$, the area growth increases due to the fact that a $\gamma_{T}$-jump of 0.8 unit is not enough to completely eliminate the chromatic non-linear effect.

Fig. 4 shows that with the $\boldsymbol{\gamma}_{\boldsymbol{T}}$-jump, the area growth is approximately a linear function of the net coupling impedance. If measures are taken so that the effective space-charge impedance is properly compensated by the inductive wall impedance, the growth will be miniruized accordingly.

\section{Tolerance on the $160 \mathrm{MHz}$ RF System}

The transition energy is crossed with the 26.7 MHz rf system using a peak voltage of $300 \mathrm{kV}$. During the crossing, measures are taken to mininize the voltage of the $160 \mathrm{MIIz}$ If system (for storage). However, due to imperfection in plase and amplitude control, a noll-zero voltage may still remain.

Previous studies ${ }^{13}$ indicate that for a given phase $\phi_{2,}$ of the $160 \mathrm{MHz}$ vollage relative to the center of the bunch, the bunch-area growth is linearly proportional to the ratio of the amplitude of this voltage $\hat{V}_{2}$ to that of the accelerating voltage. On the other hand, for a given amplitude $\hat{V}_{2}$, the most severe bunch distortion occurs when $\phi_{2 \mathrm{~s}}=180^{\circ}$. Since the bunch length is comparable to the bucket width of the $160 \mathrm{MHz}$ rf system, the bunch appears $S$-shaped after transition if the remnant voltage is significant.

Fig. 5 shows the efteclive growth of the bunch area as a function of the rennant valtage for cases of $\phi_{2},=0$ and $180^{\circ}$, respectively, calculated from the results of the computer simulation. It is observed that with the $\gamma_{T}$-jump, the additional growth in area will be less 

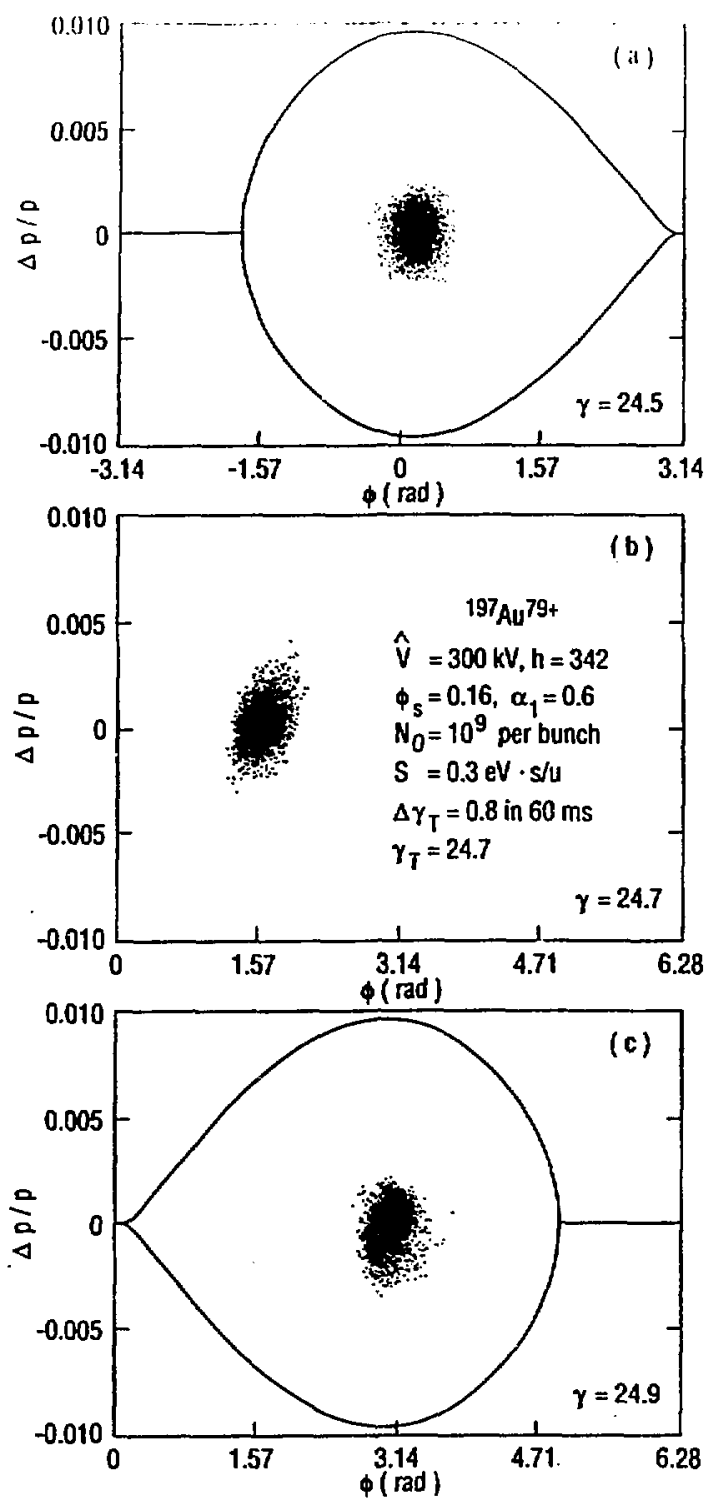

Figure 1: Longiludinal phase-space diagrams of a ${ }^{197} \mathrm{Au}^{79+}$ bunch before, al, and after transition in R.HIC at $\gamma_{s}=24.5,24.7$, and 24.9 , respeclively, nsing a $\gamma_{\Gamma}$-jump of 0.8 unil in $60 \mathrm{lls}$. 


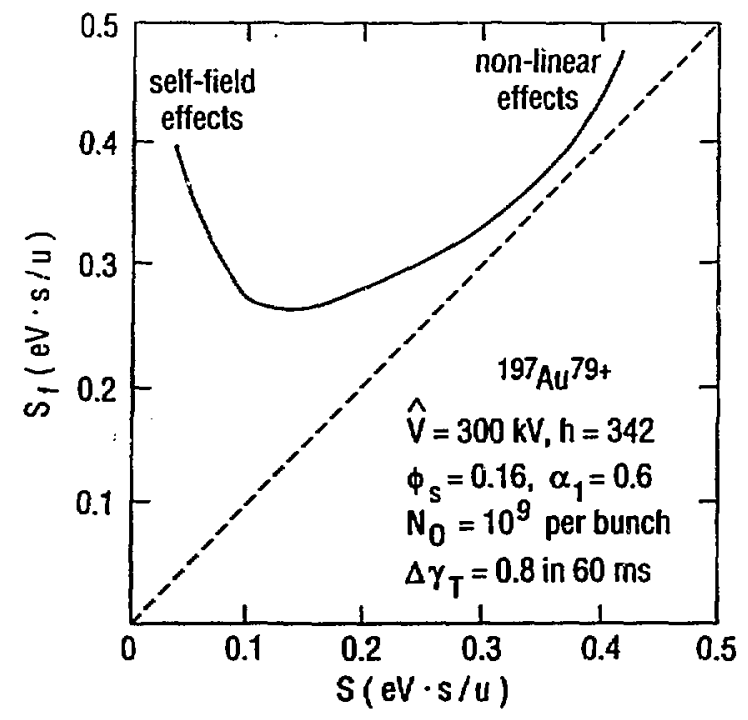

Figure 2: Longitudinal bunch area after transition as a function of the area before transition. than $10 \%$ if $\hat{V}_{2}$ is less than $10 \mathrm{kV}$.

\section{Conclusion}

Both analytical and computational studies have been performed to investigate the transition-energy crossing of different species of ion beams. With the currently proposed RHIC latlice and beam intensity, ${ }^{7}$ it is shown that a " ${ }_{T}$-jump" of aloout 0.8 unit performed in about $60 \mathrm{~ms}$, is required to achicve a crossing with no particle loss and negligible (less than $20 \%$ for all species of ions) bunch-area growth. Such a "clcan" crossing requires the amplitude of the remnant voltage of the $160 \mathrm{MIz}$ rf system to be controlled below $10 \mathrm{kV}$ during the time of transition.

\section{Acknowledgment}

The author would like to thank A.G. Ruggiero for helpful discussions, and II. Uahn for comment. 

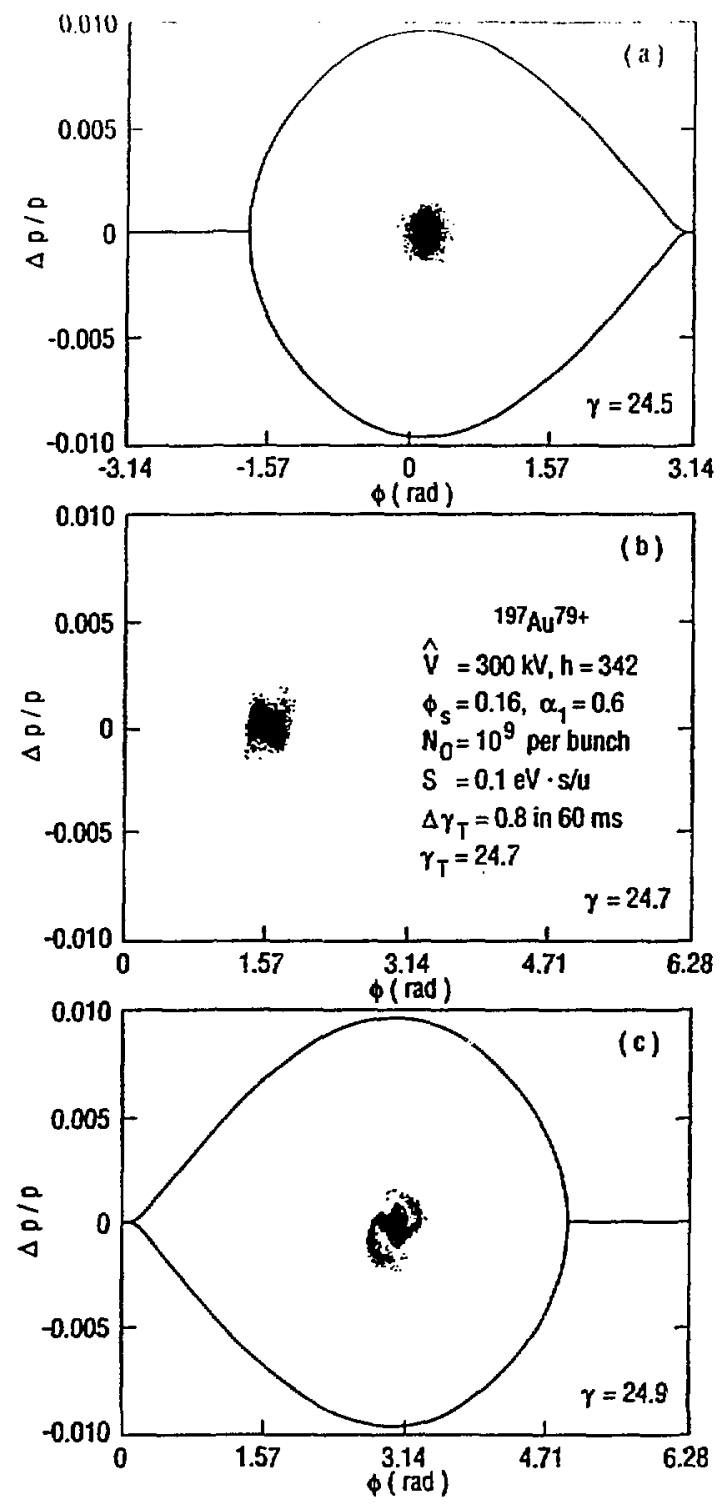

Figure 3: Transition crossing of a bunch of $0.1 \mathrm{eV} \cdot \mathrm{s} / \mathrm{u}$ arca before transition. Self-ficld mismalch and microwave instability mainly canse growth in bunch area. 


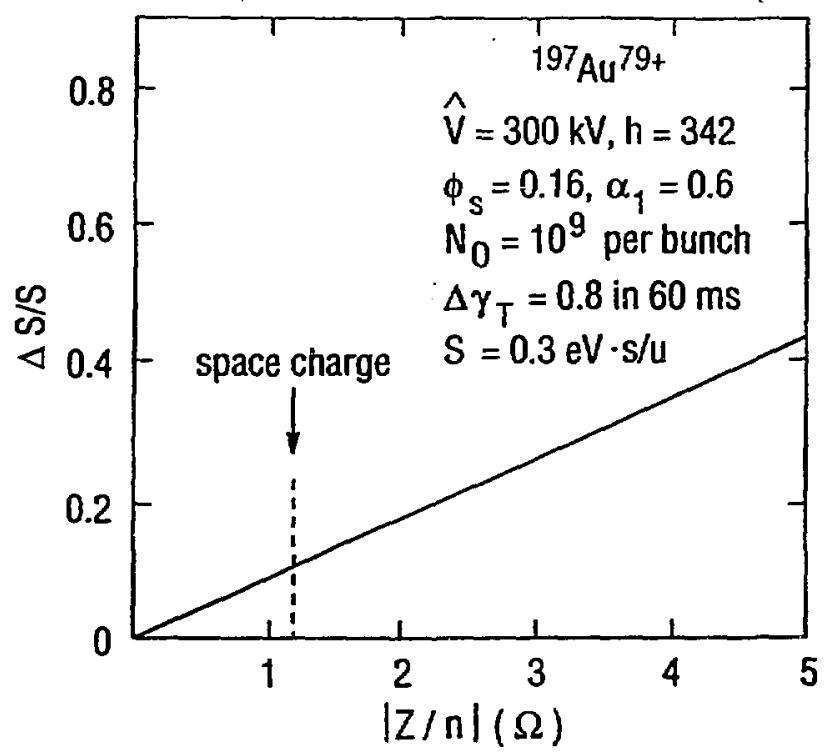

Figure 4: Growth in longitudinal bunch area as a function of the net coupling inpedance.

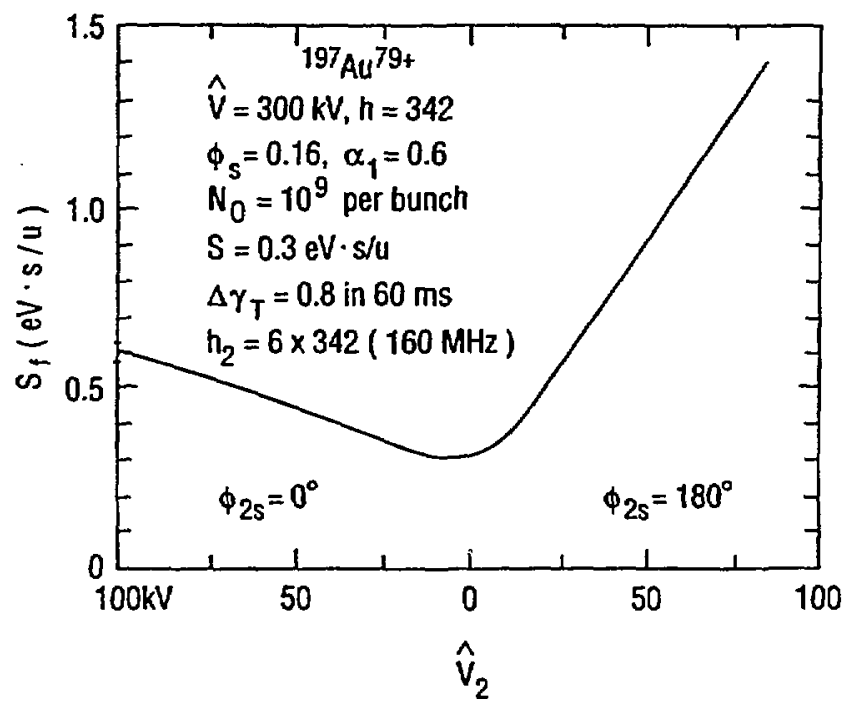

Figure 5: Longitudinal bunch area after transition as a function of the anyjlitude of the retnuant $160 \mathrm{MHz}$ voltage for $\phi_{2,}=0$ and $180^{\circ}$, respectively. 


\section{References}

1. E.D. Courant and H.S. Snyder, Ann. Pliys. 3, 1 (1958).

2. J.C. IIerrera, Particle Accelerators 3, 49 (1972).

3. S.Y. Lee and J.M. Wang, IEEE Trans. Nucl. Sci. NS-52, 2323 (1985).

4. J. Wei, Longitudinal Dynamics of the Non-Adiabatic Regime on Alternating-Gradient Synchrotrons, Ph.D thesis, State University of New York at Stony Brook, 1990.

5. K. Johnsen, Proceedings of CERN Symposium on Higl-Energy Accelerators and Pion Physics (Geneva, 1956), Vol.1, p.106.

6. S.Y. Lee and J. Wei, Proceedings of European Particle Accelerator Conference (Rome, 1988), p.764.

7. Conceplual Design of the Relativistic Heavy Ion Collider, BNL-52195 (Brookhaven National Laboratory, Upton, New York, 1989).

8. A. Sørenssen, Proceedings of 6th International Confcrence on Higlt-Energy Accelerators (Cambridge, Mass., 1967), p.474.

9. J. Wei and S.Y. Lee, Particle Accelerators 28, 77 (1990).

10. J. Wei, subnitted to Particle Accelerators.

11. J. Wci, and S.Y. Lee, Proc. Workshop on RIIIC Performance (Brookhaven Nationtal Laboratory, 1988), p.339.

12. J. Wei, Proc. Fermilab III Instabilities Workshop (Batavia, 1990), p.106.

13. S.Y. Lee, and J. Wei, Proc. 1989 Particle Accelerator Conference (Chicago, 1989), Vol. 2, 1169-1171. 